\title{
Tumor Invasion Margin on the Riemannian Space of Brain Fibers
}

\author{
Dana Cobzas ${ }^{1}$, Parisa Mosayebi ${ }^{1}$, Albert Murtha ${ }^{2}$, and Martin Jagersand ${ }^{1}$ \\ ${ }^{1}$ Department of Computer Science, University of Alberta, Canada \\ ${ }^{2}$ Department of Oncology, University of Alberta, Canada
}

\begin{abstract}
Gliomas are one of the most challenging tumors to treat or control locally. One of the main challenges is determining which areas of the apparently normal brain contain glioma cells, as gliomas are known to infiltrate for several centimeters beyond the clinically apparent lesion visualized on standard CT or MRI. To ensure that radiation treatment encompasses the whole tumour, including the cancerous cells not revealed by MRI, doctors treat a volume of brain extending $2 \mathrm{~cm}$ out from the margin of the visible tumour. This expanded volume often includes healthy, non-cancerous brain tissue.

Knowing that glioma cells preferentially spread along nerve fibers, we propose the use of a geodesic distance on the Riemannian manifold of brain fibers to replace the Euclidean distance used in clinical practice and to correctly identify the tumor invasion margin. To compute the geodesic distance we use actual DTI data from patients with glioma and compare our predicted growth with follow-up MRI scans. Results show improvement in predicting the invasion margin when using the geodesic distance as opposed to the $2 \mathrm{~cm}$ conventional Euclidean distance.
\end{abstract}

\section{Introduction}

Primary brain tumors are tumors which start from a glial cell in the nervous system. High grade variations of these tumors grow very fast often leading to a life-threatening condition. Current imaging techniques such as CT and MRI detect only the part of the tumor with a high concentration of tumor cells. The conventional medical practice is to perform maximally safe surgical resection and then irradiate the remaining tumor cells (visible and occult). The radiotherapy is conventionally applied to a margin of about $2 \mathrm{~cm}$ around the visible tumor which is a very rough approximation of the probable location of tumor cells. This approach does not consider tumor growth dynamics in different brain tissues, thus it may result in killing some healthy cells while leaving alive cancerous cells in other areas. These cells may cause re-occurrence of the tumor later in time which limits the effectiveness of the therapy.

To improve the therapeutic outcome, more accurate prediction of the tumor invasion margin is necessary. Based on the generally accepted belief that glioma cells preferentially spread along nerve fibers [1, we propose here a new approach of computing the tumor invasion margin that makes use of a geodesic distance 
defined on a manifold of brain fibers. This formulation is very easily transferable to radiation therapy software by replacing the uniform (Euclidean) distance currently used to define the $2 \mathrm{~cm}$ invasion margin (that will be radiated) with the geodesic distance.

Many efforts have been made to mathematically model the Glioma tumor growth. Following 2] these approaches are classified in three major categories: microscopic, mesoscopic and macroscopic. Micorscopic models describe the growth process in sub-cellular level, concentrating on activities that happen inside the tumor cell. Mesoscopic approaches focus on interactions between tumor cells and their surrounding tissue while macroscopic approaches focus on tissue level processes considering macroscopic quantities such as tumor volume and flow. As we are interested in modeling tumor invasion, we will restrict our discussion to macroscopic models.

Most models on macroscopic tumor growth use a reaction-diffusion term based on diffusion equation introduced by Murray 3 . Swanson et al. 4 used this term to generate a model assuming different motility of tumor cells in gray and white matter. They further enhanced their model to simulate the virtual gliomas 5 . However, this is an isotropic model which only simulates high grade glioma while low-grade gliomas exhibit complex shapes and are not well simulated by an isotropic model. More recent approaches use anisotropic diffusion along white matter fibers as given by the diffusion tensors (from Diffusion Tensor Images-DTI) to simulate more complex tumors. With limited availability of DTI data, existing techniques simulate growth based either on atlas tensors registered with the patient [6] or tensors from a healthy subject [7] unregistered with the patient. Recently a mechanical model of the mass effect was added to the reaction diffusion equation 6 6] resulting in a more physically plausible growth simulation.

In this paper, we introduce a novel model more directly driven by the particular patient DTI data to predict the tumor invasion margin using the geodesic distance defined on the Riemannian manifold of brain. The formulation of white matter as a Riemannian manifold was first introduced by O'Donnell et al. [9] and later formalized by Lenglet et al. 10. They used this model for white matter connectivity mapping (tractography). Our geodesic growth model is concerned with predicting only the current tumor spread (invasion) that is not visible in the MRI images for better radiation therapy planning and therefore doesn't include mass effect. Konukoglu et. al [1] has previously used the diffusion equation to find tumor invasion margin but their method was tested only on synthetically grown tumors. Our geodesic model can more easily be incorporated into radiation planning software that already makes use of a distance (Euclidean) in defining the target region.

In addition to introducing the geodesic distance in the tumor growth concept, another contribution of the paper is the application of the method on actual patient specific DTI data. Furthermore, our method takes into account natural barriers to glioma growth such as the skull, the tentorium cerebelli and the falx cerebri. We tested our model on eight different patients by growing the tumor on the DWI scan of the patient and comparing the predicted distance with real 
growth shown on later MRI scans of the same patient. Comparative results of using geodesic distance show an improvement vs. uniform (Euclidean) distance.

\section{Material and Methods}

\subsection{Tumor Invasion Using Geodesic Distance on Brain Fibers Manifold}

The brain tumor infiltrating component can be mathematically modeled using anisotropic diffusion 76 .

$$
\frac{\partial c}{\partial t}=\nabla \cdot(D \nabla c)=\mathcal{L} c
$$

where $c$ is the normalized concentration of tumor cells and $D$ is the diffusion tensor of the tumor cells. Knowing that tumor diffusion is similar to water diffusion [1, $D$ can be replaced with the diffusion tensor obtained from diffusion tensor imaging.

Tumor grows with different speed in white vs gray matter (with a factor of about $\alpha=10$ (4). While ideally this should be directly reflected by the diffusion tensors $(D T)$ magnitude, due to noise and discretization problems and the fact that tumor might grow at a different speed than water diffusion, $D \neq D T$. One could estimate a function $D(x)=C(x) D T(x)$ where $C(x)$ is a matrix representing a spatial transform function. However $C(x)$ is intractable to estimate from limited data. Instead, after experimenting with real patient DTI data, we found that the linear weights $w(x)=\alpha F A(x)$ produce good results. $F A$ represents the fractional anisotropy computed from tensor data.

The diffusion tensors in white matter are anisotropic, indicating the direction of fibers. As a consequence the anisotropic diffusion growth model would encourage diffusion of cancer cells along fibers [1. We propose the use of a geodesic distance on the Riemannian manifold of white matter fibers to model the anisotropic tumor growth. O'Donnell et al [9] and Lenglet et al. 10 introduced the formulation of the white matter as a Riemannian manifold characterized by the infinitesimal anisotropic diffusion operator $\mathcal{L}$. They made the link between the diffusion tensor data $D$ and the white matter manifold geometry and showed that the diffusion operator can be associated with a metric $G=D^{-1}$. This metric allows computation of geodesic path and distances between points on the brain and was previously used for fiber connectivity.

Geodesic Distance Calculation. Following [10, the distance $\Phi$ from a nonempty closed subset $K$ is found by solving the eikonal equation on the 3dimensional Riemannian manifold $(M, g)$ (connected and complete)

$$
\left\{\begin{array}{l}
|\operatorname{grad} \Phi|=1 \quad \text { in } \quad M \backslash K \\
\Phi(x)=\Phi_{0}(x) \text { for } \quad x \in K
\end{array}\right.
$$

where $\Phi_{0}(x)=0 \quad \forall x \in K$. 


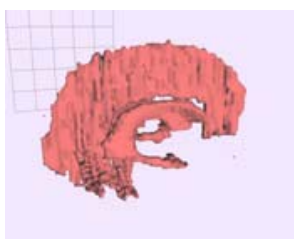

(a) Barriers

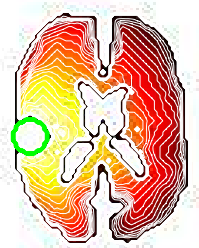

(b) Geod. dist.

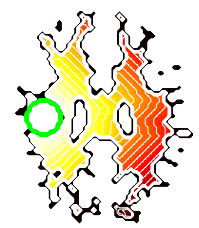

(c) Geod. dist. on white matter

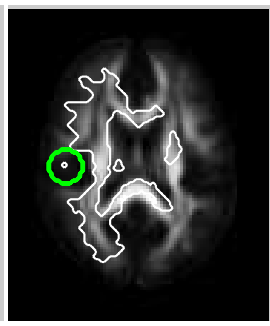

(d) Geod. dist. isocontour on FA

Fig. 1. The result of applying the geodesic distance model to a DTI atlas. Colors show the geodesic distance from the initial position.

Representing the distance function $\Phi$ as the zero levelset of a signed distance function $\Psi, \Psi(x, t)=0 \Leftrightarrow t=\Phi(x)$, Equation 2 can be reformulated as finding $\Psi$ the viscosity solution of

$$
\left\{\begin{array}{l}
\frac{\partial \Psi}{\partial t}+|\operatorname{grad} \Psi|=0 \forall t>0 \\
\Psi(x, 0)=\Psi_{0}(x)
\end{array}\right.
$$

where $\Psi_{0}$ is the signed distance function of $\Phi_{0}$.

To numerically solve hyperbolic Hamilton-Jacobi Equation 3 we approximated the continuous flux $|\operatorname{grad} \Psi|^{2}$ as [10]:

$$
\begin{aligned}
|\operatorname{grad} \Psi|^{2}= & \sum_{i=1}^{3} g^{i i}\left(\max \left(D_{x_{i}}^{-} \Psi, 0\right)^{2}+\min \left(D_{x_{i}}^{+} \Psi, 0\right)^{2}\right)+ \\
& \sum_{i \neq j} g^{i j} \operatorname{minmod}\left(D_{x_{i}}^{+} \Psi, D_{x_{i}}^{-} \Psi\right) \min \bmod \left(D_{x_{j}}^{+} \Psi, D_{x_{j}}^{-} \Psi\right)
\end{aligned}
$$

where $g_{i, j=1 \ldots 3}^{i j}$ are components of the inverse matrix $G^{-1}, D_{x_{i}}^{ \pm} \Psi$ are upwind approximation of the gradient of $\Psi$ in $x_{i}$ and $\operatorname{minmod}(a, b)=\min (a, 0)+$ $\max (b, 0)$.

Geodesic Distance for Tumor Growth Prediction. When using the geodesic distance in the context of growth prediction, we chose as the origin of the grwoth (subset $K$ ) the visible tumor margin. In addition, as the brain contains several obvious natural barriers to glioma growth such as the skull, ventricular system, the tentorium cerebelli and the falx cerebri, $M$ is defined as the brain volume that doesn't contain those barriers. Fig. 1(a) shows an example of segmented barriers (ventricles, falx, tentorium). Fig. 1(b-d) shows examples of geodesic distance computed on the ICBM DTI-81 atlas [12]: (b) shows the geodesic distance computed with linear tensor weighting that originates from a sphere (green circle in the figure) until reaches the skull boundary. (c) shows the geodesic distance computed only in the white matter tensors instead of the whole brain tensors; (d) shows an isocontour of the geodesic distance aligned with FA values. Notice how the distance follows the fiber directions. 


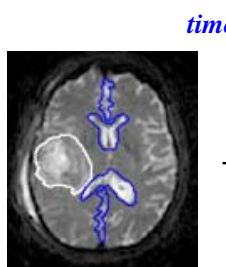

DWIO

(a) initial segm. barriers time1

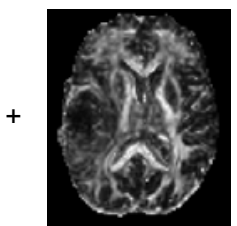

(b) weights

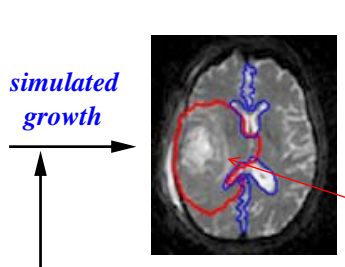

(c) $\begin{aligned} & \text { geodesic } \\ & \text { distance }\end{aligned}$

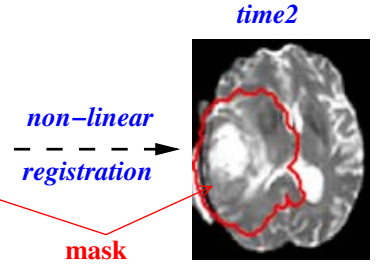

warped DWIO

(d) and geod. dist.

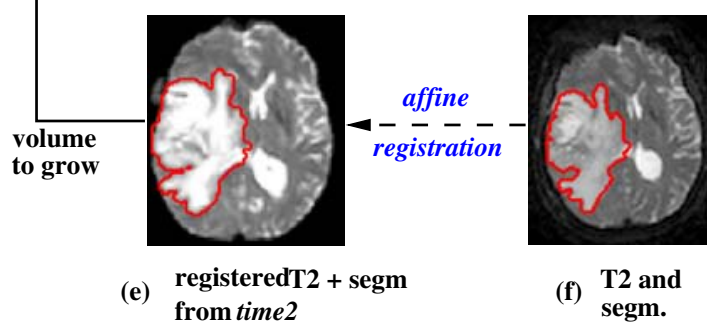

Fig. 2. Overview of validation system. Tumor growth is simulated on DTI data from time1 and compared with the manual segmentation on data at time2.

\section{$2.2 \quad$ Patients and Data}

We used MRI and DTI data from clinical scans of patients with GBM 11 Each patient has a pre-RT MRI scan. Follow-up DTI or MRI scans have been made after treatment at intervals of about 3-6 months. DTI data has a resolution of $128 \times 128 \times 60$ while MRI data has a resolution of $512 \times 512 \times 21$. Typically at least one or two DTI data is acquired after radiation and the rest of the scans are conventional MRI images (T2, T1, FLAIR). To minimize the effects of radiation treatment not accounted in our model, we use data from the first DTI scan acquired after radiation for estimating the diffusion-based invasion margin and compare our model with the actual growth observed in later MRI-T2 scans.

Both the tumor growth and the comparison is done based on segmentations of the high signal region adjacent to the gross tumor on MRI-T2 or DWI0 (the DWI scan with zero b-value similar to T2). This region contains tumor, associated edema and microscopic tumor cell infiltration. Segmentations are done using a semi-automatic tool developed in our lab. Growth barriers (ventricular system, falx cerebri and tentorium cerebelli) are manually delineated using the same software. An expert radiation oncologist validated all segmentations.

\subsection{Data Processing and Validation Procedure}

Fig. 2 shows an overview of the growth validation system. We grow the tumor from time1 to approximately its size at time2 and then compare the result of our model with the actual growth. This validation assumes that the visible growth

\footnotetext{
${ }^{1}$ The data collection protocol was approved by REB and the patients that have pro-
} vided an informed consent. 
Table 1. Jaccard (overlap) scores for comparing registered ground truth with geodesic and Euclidean growth. $\operatorname{Jaccard}(A, B)=(A \cap B) /(A \cup B) \operatorname{Hausdroff}(A, B)=$ $\max \left\{\sup _{a \in A} \inf _{b \in B} d(a, b), \sup _{b \in B} \inf _{a \in A} d(a, b)\right\}$.

\begin{tabular}{|l|cccc|c|cccc|c|}
\hline & \multicolumn{4}{|c|}{ Jaccard score (\%) } & \multicolumn{4}{c|}{ Hausdorff distance $(\mathrm{mm})$} \\
\cline { 2 - 11 } & 1 & 2 & 3 & 4 & mean & 1 & 2 & 3 & 4 & mean \\
\hline Geodesic dist. & 65 & 75 & 72 & 65 & 69.2 & 9.32 & 7.68 & 8.90 & 16.72 & 10.65 \\
Euclidean dist. & 59 & 65 & 64 & 60 & 62.0 & 10.02 & 8.60 & 9.38 & 17.49 & 11.37 \\
\hline
\end{tabular}

in the subsequent times occurs over the invisible but already-infilterated regions at the initial time. We use the first or second DTI scan after treatment to generate growth starting from the manually segmented visible high signal on DWI0 (Fig. 2 (a) - edema, tumor swelling - time1). We extract diffusion tensors using ExploreDTI [13] that are then weighted (Fig. 2(b)) based on the Fractional Anisotropy values. Tumor growth is simulated by iteratively solving Eq. 3 .

We validated growth by comparing the results from the geodesic distance (Fig. 2 (c)) with manually segmented high signal on T2/DWI0 from later follow up scans (Fig. 2 (f) - time2). This comparison requires registration. We used affine registration of T2-MRI/DWI0 data from time2 with the DTI (DWI0) data at time 1 to determine the approximate growth volume used as a stopping criterion for the geodesic distance simulation. We also used the same registration for visual comparisons (comparing (c) with (e) - see Fig. 3). For fair comparison between Euclidean and geodesic distance, we apply the same process also for the Euclidean distance instead of simply growing the tumor to the $2 \mathrm{~cm}$ margin.

The linear registration doesn't take into account the mass effect as a consequence of tumor growth from time1 to time2. This is easily noticed in Fig. 2 (e) which shows the result of affine registration: the growth affected ventricle shape in time2 is incorrectly registered with data at time1. Therefore for numerical scores we applied non-linear registration of DWI0 data and the predicted geodesic growth from time1 to T2-MRI data and segmented edema at time2. For correct non-linear registration we masked edema label (time2) and the generated growth label (time1). Those regions contain abnormalities and they cannot be taken into account for the registration score. Fig. 2 (d) shows the result of nonlinear registration that can now be compared with (f). Now the shape of the ventricles correctly aligns on the space of time2. For both registrations we used FSL tools 14] (FLIRT for linear and FNIRT for non-linear registration).

\section{Results}

Our dataset includes 24 DWI but we could only use the 8 data that showed tumor growth after treatment. We applied the growth models with the data processing explained in Section 2.3 to each patient data. Fig. 3 (2) shows the comparative results of real growth with geodesic and Euclidean growth. The results show that where the tensor values are less noisy, the geodesic distance model can track the path of fibers and therefore matches tumor growth, as opposed to when using 

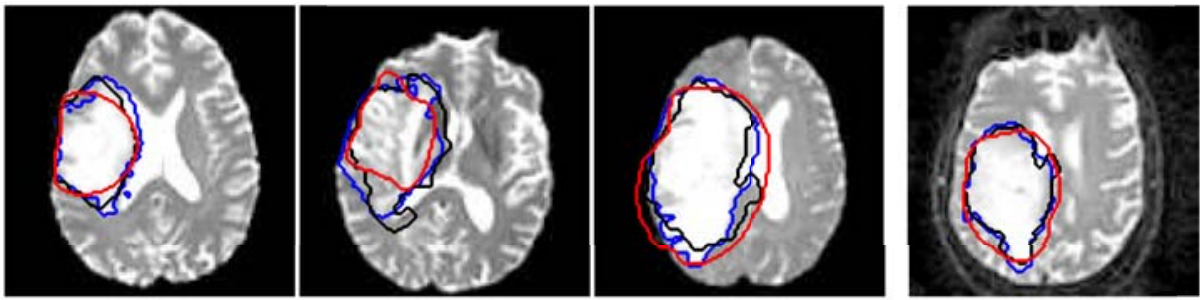

Fig. 3. (1) Comparative results on time2 DWI0 corresponding to Table 1 Patient 1-4 (left-right). Blue line shows manual segmentation, black line shows simulated geodesic growth and red line shows simulated Euclidean growth.
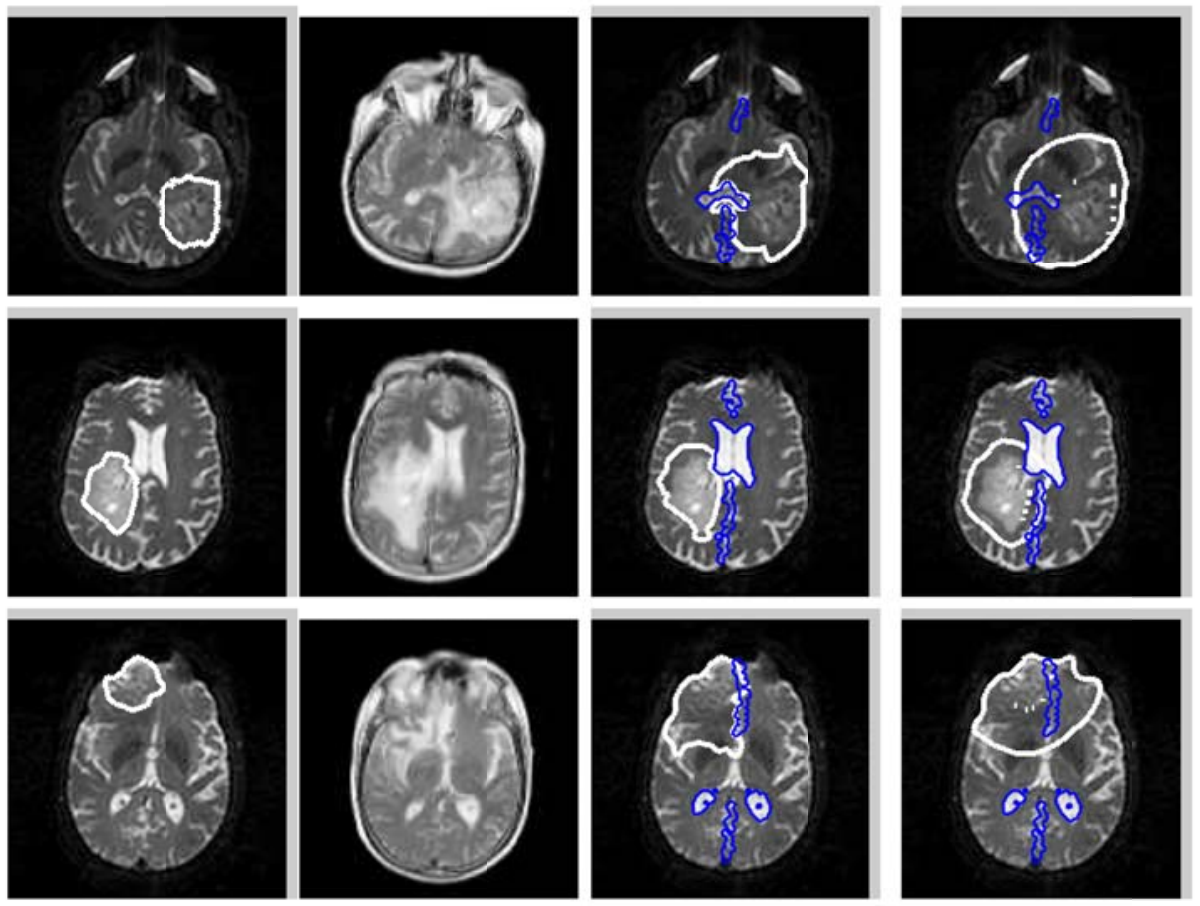

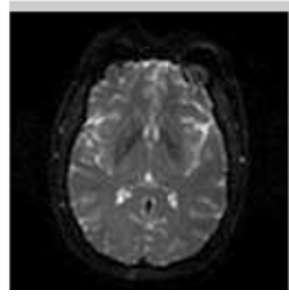

(a) Patient at time1

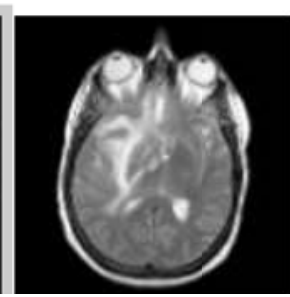

(b) Patient at time2

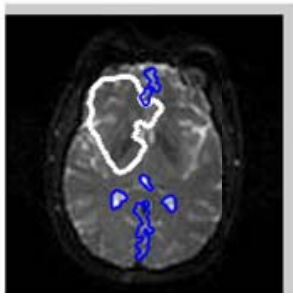

(c) Geod. dist.

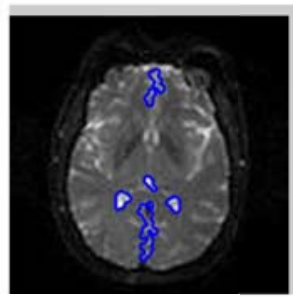

(d) Euclid. Dist.

Fig. 3. (2) Comparative results for different patients of Geodesic (c) and Euclidean (d) simulated growth starting from segmented tumor at time1 (a) and linearly registered followed up scans at time2 (MRI-T2 or DWI) (b). Barriers are shown in blue. 
the Euclidean model. Notice how in the example from the last row of Fig. 3 the Euclidean distance has not reached the shown tumor slice while the geodesic distance correctly models the growth.

To numerically compare our model with the conventional Euclidean model, we calculated the Jaccard scores and Haudorff distances shown in Table 1. Corresponding visual results are shown in Fig. 3 (1). As mentioned in Section 2.3 for reducing the mass effect we applied non-linear registration to warp data from time1 into the space of time2. Due to registration problems we could only calculate scores for 4 patients. Numerical results show an improvement of about $5-10 \%$ for simulated growth using the geodesic distance compared to the Euclidean distance. This difference correspond to millions of saved brain cells.

\section{Discussion}

In this paper, we introduced the use of geodesic distance on the Riemannian manifold of brain fibers to detect the glioma brain tumor invasion margin. The model was tested on several real patients data and a DTI atlas. In contrast to most of the previous works in this area, we used real tensors of the patients obtained by the standard clinical procedure instead or registered atlas tensors. Comparative results between real growth in follow up scans and simulated growth based on geodesic and Euclidean distance prove that the use of the geodesic distance could significantly improve radiation therapy treatment.

To further improve results on noisy clinical data we plan to apply better tensor extraction and regularization. Furthermore, although the non-linear registration used between time1 and time2 to numerically validate results solves the problem of mass effect to a good extent, it cannot solve it completely. Hence, the error in the non-linear registration produces inaccuracies. For better non-linear registration in the presence of mass effect we plan to incorporate a mechanical model into the registration (similar to [15]).

\section{References}

1. Kuroiwa, T., Ueki, M., Chen, Q., Suemasu, H., Taniguchi, I., Okeda, R.: Biomechanical characteristics of brain edema: the difference between vasogenic-type and cytotoxic-type edema. Acta Neurochir. Suppl. 60, 158-161 (1994)

2. Hatzikirou, H., Deutsch, A., Schaller, C., Simon, M., Swanson, K.: Mathematical modelling of glioblastoma tumor developement: A review. Mathematical Models and Methods in Applied Sciences 15(11), 1779-1794 (2005)

3. Murray, J.: Mathematical Biology. Springer, Heidelberg (1989)

4. Swanson, K., Alvord, E.J., Murray, J.: A quantitative model for differential motility of gliomas in grey and white matter. Cell Proliferation 33, 317-329 (2000)

5. Swanson, K., Alvord, E., Murray, J.: Virtual brain tumors (gliomas) enhance the reality of medical imaging and highlight inadequacies of current therapy. British Journal of Cancer 85, 14-18 (2002) 
6. Clatz, O., Sermesant, M., Bondiau, P.Y., Delingette, H., Warfield, S.K., Malandain, G., Ayache, N.: Realistic simulation of the 3d growth of brain tumors in MR images coupling diffusion with mass effect. IEEE Transactions on Medical Imaging 24(10), 1334-1346 (2005)

7. Jbabdi, S., Mandonnet, E., Duffau, H., Capelle, L., Swanson, K., Pelegrini, M., Guillevin, R., Benali, H.: Simulation of anisotropic growth of low-grade gliomas using dif. tensor imag. Magnetic Resonance in Medicine 54, 616-624 (2005)

8. Hogea, C., Davatzikos, C., Biros, G.: Modeling glioma growth and mass effect in 3D MR images of the brain. In: Ayache, N., Ourselin, S., Maeder, A. (eds.) MICCAI 2007, Part I. LNCS, vol. 4791, pp. 642-650. Springer, Heidelberg (2007)

9. O'Donnell, L., Haker, S., Westin, C.-F.: New approaches to estimation of white matter connectivity in diffusion tensor MRI: Elliptic PDEs and geodesics in a tensor-warped space. In: Dohi, T., Kikinis, R. (eds.) MICCAI 2002. LNCS, vol. 2488, p. 459. Springer, Heidelberg (2002)

10. Lenglet, C., Deriche, R., Faugeras, O.: Inferring white matter geometry from diffusion tensor MRI: Applic. to connectivity mapping. In: MICCAI (2004)

11. Konukoğlu, E., Clatz, O., Bondiau, P.-Y., Delingette, H., Ayache, N.: Extrapolating tumor invasion margins for physiologically determined radiotherapy regions. In: Larsen, R., Nielsen, M., Sporring, J. (eds.) MICCAI 2006. LNCS, vol. 4190, pp. 338-346. Springer, Heidelberg (2006)

12. LONI: atlases, http://www.loni.ucla.edu/atlases/

13. ExploreDTI, http://www.exploredti.com/

14. FSL: tools, http://www.fmrib.ox.ac.uk/fsl/

15. Mohamed, A., Zacharaki, E., Shen, D., Davatzikos, C.: Deformable registration of brain tumor images via a statistical model of tumor-induced deformation. Medical Image Analysis 10(5), 752-763 (2006) 